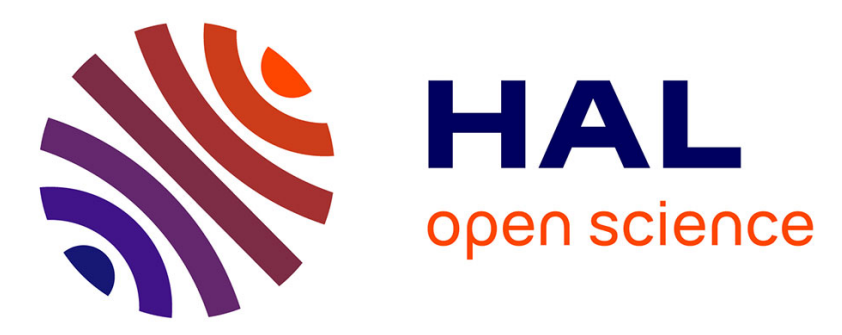

\title{
On the speed of solitary waves running down a vertical wall
}

\author{
C. Ruyer-Quil, Paul Manneville
}

\section{To cite this version:}

C. Ruyer-Quil, Paul Manneville. On the speed of solitary waves running down a vertical wall. Journal of Fluid Mechanics, 2005, 531, pp.181-190. 10.1017/s0022112005003885 . hal-01024966

\section{HAL Id: hal-01024966 \\ https://hal-polytechnique.archives-ouvertes.fr/hal-01024966}

Submitted on 28 Jul 2014

HAL is a multi-disciplinary open access archive for the deposit and dissemination of scientific research documents, whether they are published or not. The documents may come from teaching and research institutions in France or abroad, or from public or private research centers.
L'archive ouverte pluridisciplinaire HAL, est destinée au dépôt et à la diffusion de documents scientifiques de niveau recherche, publiés ou non, émanant des établissements d'enseignement et de recherche français ou étrangers, des laboratoires publics ou privés. 


\title{
On the speed of solitary waves running down a vertical wall
}

\author{
By C. RUYER-QUIL ${ }^{1}$ AND P. MANNEVILLE \\ ${ }^{1}$ Laboratoire FAST - UMR CNRS 7608, Campus Universitaire, 91405 Orsay, France \\ ${ }^{2}$ LadHyX - UMR CNRS 7646, École Polytechnique, 91128 Palaiseau, France
}

(Received 5 October 2004 and in revised form 1 February 2005)

Solitary-wave solutions to surface equations or two-equation models of film flows are investigated within the framework of dynamical system theory. The limiting behaviour of one-humped solitary waves (homoclinic orbits) at large Reynolds numbers is considered. Their predicted speed is in good agreement with numerical findings. The theory also explains the absence of solitary-wave solutions to the Benney equation in the same limit.

\section{Introduction}

Due to the widespread use of film flows in industrial applications, the stability of thin-film flows has received much attention, starting with the seminal work by Kapitza (Kapitza 1948; Kapitza \& Kapitza 1949). From a theoretical viewpoint, the interest of this system stems from the fact that the primary instability is spanwiseindependent (Yih 1955), of long-wavelength, and supercritical. In most relevant flow regimes, the flow remains close to that of the flat-film solution, called the Nusselt flow, with thickness $h=h_{\mathrm{N}}$ and parabolic velocity profile.

We focus here on liquid films flowing along vertical walls. The usual control parameters are then just the Reynolds number $R=g h_{\mathrm{N}}^{3} / 3 v^{2}$ and the Weber number $W=$ $\sigma / \rho g h_{\mathrm{N}}^{2}$, comparing inertia to viscous effects, and surface tension to gravity, respectively. Here $g$ is the gravity acceleration and $\rho, v$ and $\sigma$ are the fluid's density, kinematic viscosity and surface tension. The proximity to the Nusselt flow is measured by the so-called film parameter $\epsilon$ scaling the typical slope of the film. In flow regimes of interest, the cross-stream coherence of the flow is ensured by viscosity whereas the slope is maintained small enough thanks to surface tension effects. For thickness fluctuations with wavelength $\ell$, the order of magnitude of this parameter can be obtained through the estimate $\rho g \sim \sigma \partial_{x x x} h$ as $h_{\mathrm{N}} / \ell \sim W^{-1 / 3}$. Following Shkadov (1977), it is then advisable to rescale the streamwise and cross-stream directions $x$ and $y$ differently, in order to make this slope of order unity, hence defining the scale ratio $\kappa=W^{-1 / 3}$. In this process the Reynolds number $R$ is replaced by $\delta=3 R / \kappa$ that compares inertia to surface tension and viscosity directly. (The reduced Reynolds number originally introduced by Shkadov was $\delta / 45$ due to different numerical scaling choices.) A second parameter $\eta=W^{-2 / 3}$ measuring the intensity of the streamwise viscous dispersion is then substituted for the Weber number. When surface tension is strong, the Weber number is large, so that $\kappa$ and $\eta$ are small, making typical instability wavelengths long and keeping viscous dispersion negligible.

The film's dynamics is essentially that of isolated large-amplitude solitary waves in the form of a main hump preceded by smaller capillary ripples, which travel much 
faster than linear waves (Alekseenko, Nakoryakov \& Pokusaev 1994; Liu \& Gollub 1994). Direct simulation of Navier-Stokes equations with a free surface remains a formidable task, see e.g. Malamataris et al. (2002). This difficulty motivated the search for reliable reduced models. The smallness of parameter $\epsilon$ allows a drastic simplification of the primitive equations (Shkadov 1967, 1977) which, after elimination of the pressure, yields the so-called boundary-layer (BL) equations. However this simplification is still not sufficient since these equations retain the same physical dimensionality as the primitive equations, which focuses the attention on even more simplified models where the cross-stream dependence is eliminated through averaging and/or long-wave expansion.

\subsection{Surface equations}

By performing a gradient expansion of the set of primitive equations, Benney (1966) obtained a single evolution equation for the film thickness which, using Shkadov's notations, is

$$
\partial_{t} h+\frac{1}{3} \partial_{x}\left\{h^{3}+\frac{2}{35} \delta \partial_{x}\left(h^{7}\right)+h^{3} \partial_{x x x} h\right\}=0 .
$$

Benney's equation is the prototype of so-called surface equations, solely involving the local film thickness $h(x, t)$ and its derivatives. They are derived by integrating the (exact) continuity equation across the fluid layer:

$$
\partial_{t} h+\partial_{x} q=0,
$$

where $q=\int_{0}^{h} u \mathrm{~d} y$ is the local flow rate. Approximations enter when truncated expressions for $q$, obtained e.g. through a long-wavelength expansion, are inserted in (1.2).

Numerical simulations of (1.1) demonstrated the occurrence of non-physical blowups of unsteady solutions at finite time and sufficiently large $\delta$ (Pumir, Manneville \& Pomeau 1983; Rosenau, Oron \& Hyman 1992). Ooshida (1999) however showed that the long-wavelength expansion could be regularized by applying techniques inspired from the Padé approximation method, which lead him to

$$
\partial_{t} h+\frac{1}{3} \partial_{x}\left\{h^{3}-\delta\left[\frac{2}{7} \partial_{t}\left(h^{5}\right)+\frac{36}{245} \partial_{x}\left(h^{7}\right)\right]+h^{3} \partial_{x x x} h\right\}=0 .
$$

Comparing inertial terms (with factor $\delta$ ) of (1.3) with that in (1.1), one can see the introduction of a new term involving a time derivative and a change of the coefficient of the original term from $2 / 35$ to $-36 / 245$. Ooshida's equation does not exhibit finitetime blow-up and solitary-wave solutions can be obtained for all $\delta$ but the predicted amplitudes and speeds differ from the observed values by a factor of order $2-3 . \dagger$

\subsection{Two-equation models}

The validity of the formal expansion leading to (1.1) is restricted to $\delta \ll 1$, as derived from the value of $\epsilon$ estimated from the cut-off wavenumber $\propto \sqrt{R / W}$, which yields $\epsilon R \sim R^{3 / 2} / W^{1 / 2} \sim \delta^{3 / 2}$ (Ooshida 1999). That expansion further assumes that the velocity field remains strictly enslaved to the evolution of the thickness of the film. Surface equations are thus not expected to describe wave motions at moderate $\delta$ accurately. An alternative to the single-equation approach was proposed in the seminal work by Kapitza (1948) and later by Shkadov (1967). Assuming that the velocity profile across the fluid layer remains parabolic in the wavy regime and averaging the momentum equation across the film, keeping terms up to order $\epsilon$ along with the dominant surface

$\uparrow$ The equation originally derived by Ooshida contained an extra term $-\eta \partial_{x}\left(h^{2} \partial_{x t} h\right)$ that accounts for streamwise dissipative effects, but was later shown to have little effect on the amplitude and speed of the solitary waves (Ruyer-Quil \& Manneville 2004). 
tension term, Shkadov obtained

$$
\delta \partial_{t} q=h-3 \frac{q}{h^{2}}+\delta\left[\frac{6}{5} \frac{q^{2}}{h^{2}} \partial_{x} h-\frac{12}{5} \frac{q}{h} \partial_{x} q\right]+h \partial_{x x x} h,
$$

closing the system in $h$ and $q$ by adding the mass conservation equation (1.2). Shkadov's model (1.2), (1.4) does not exhibit non-physical blow-ups of its solutions but, whereas it works quite well for vertical walls, when adapted to moderately inclined planes it leads to an incorrect prediction for the instability threshold. A refined derivation based on a systematic weighted-residual expansion of the velocity field on a polynomial basis led us later to overcome this limitation (Ruyer-Quil \& Manneville 2000). An equation similar to (1.4) was obtained but with slightly different coefficients

$$
\delta \partial_{t} q=\frac{5}{6} h-\frac{5}{2} \frac{q}{h^{2}}+\delta\left[\frac{9}{7} \frac{q^{2}}{h^{2}} \partial_{x} h-\frac{17}{7} \frac{q}{h} \partial_{x} q\right]+\frac{5}{6} h \partial_{x x x} h .
$$

When compared to equations (1.1) and (1.3), models (1.2), (1.4) and (1.2), (1.5) account for inertial effects in a clearly novel way since the local flow rate $q(x, t)$ now has its own dynamics instead of being enslaved to $h(x, t)$.

\subsection{One-humped solitary waves}

Experimentally observed solitary waves can travel without deformation at constant speed for large distances. Such solutions are computed by changing to a moving frame with coordinate $\xi=x-c t$, which transforms the partial differential problem into an ordinary differential problem. Applying this to (1.1) or (1.3) immediately leads to a single fourth-order differential equation which can be integrated once, yielding a three-dimensional dynamical system. Within the two-equation formulation, the same result is obtained but in two steps. First the mass conservation equation (1.2) becomes $-c h^{\prime}+q^{\prime}=0$, where primes denote derivatives with respect to the moving coordinate $\xi$. This equation can be integrated to yield

$$
q=c h+q_{0},
$$

where $q_{0}=\int_{0}^{h}(u-c) \mathrm{d} y$ is an integration constant corresponding to the flow rate in the moving frame. Next the second equation (1.4) or (1.5) is transformed. In all cases, the following equation is obtained:

$$
\frac{1}{3} h^{3} h^{\prime \prime \prime}+\delta \mathscr{G}(h, c) h^{\prime}+\frac{1}{3} h^{3}-c h-q_{0}=0 .
$$

In practice, $\mathscr{G}$ - to be specified below - contains all the inertial effects (with factor $\delta$ ), while the third-order derivative arises from surface tension effects. The integration constant $q_{0}$ can be fixed by imposing $h \equiv 1$ as a solution to (1.7) since $h(\xi)=H$ constant, often taken equal to the unperturbed film thickness $h_{\mathrm{N}}$, is indeed a solution to the problem. Making the changes $h \mapsto H h, c \mapsto C c, q \mapsto Q q$, preserves the structure of the equation provided that $\xi$ is also rescaled as $\xi \mapsto \Xi \xi$ and the control parameter $\delta$ as $\delta \mapsto \Delta \delta$. By substitution one is then led to $\Xi=H^{1 / 3}$ and $\Delta=H^{-11 / 3}$, whereas $C=H^{2}$ and $Q=H^{3}$. Measuring $h$ in units of $H$, i.e. with the reference unperturbed solution corresponding to $h \equiv 1$, leads to

$$
q_{0}=1 / 3-c,
$$

which will be assumed in the following. Our starting point will thus be

$$
\frac{1}{3} h^{3} h^{\prime \prime \prime}+\delta \mathscr{G}(h, c) h^{\prime}+\mathscr{H}(h, c)=0,
$$

where

$$
\mathscr{H}(h, c) \equiv \frac{1}{3} h^{3}-c h-q_{0}=\frac{1}{3}(h-1)\left(h^{2}+h+1-3 c\right),
$$



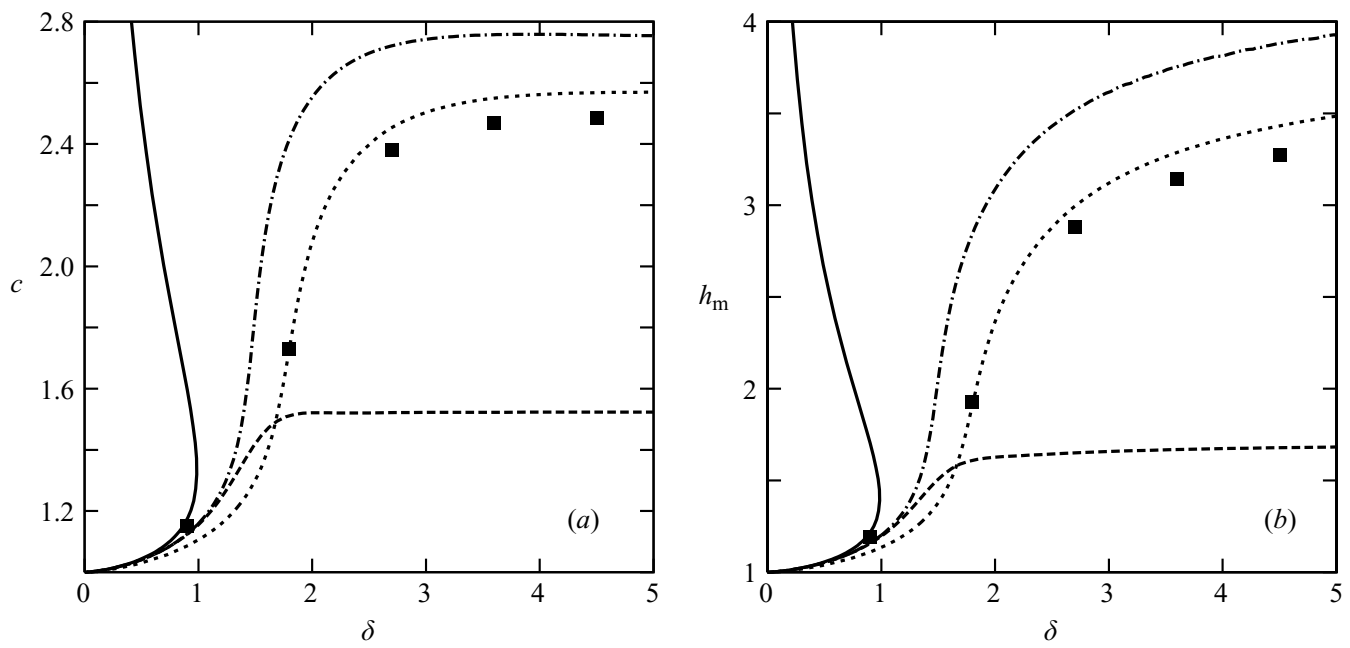

FIGURE 1. $(a)$ Speed $c$ and $(b)$ maximum height $h_{\mathrm{m}}$ as functions of the reduced Reynolds number $\delta$ for the one-hump homoclinic solutions to the Benney equation (solid line), and to the Ooshida equation (dashed line). Dotted and dash-dotted lines correspond to the Shkadov model (1.2), (1.4) and to the modified model (1.2), (1.5), respectively. Filled squares are from simulations of the BL equations (Chang et al. 1996).

and the functions $\mathscr{G}(h, c)$ corresponding to the different cases are

Benney equation (1.1): $\quad \mathscr{G}(h, c)=\frac{2}{15} h^{6}$,

Ooshida equation (1.3): $\quad \mathscr{G}(h, c)=\frac{10}{21} c h^{4}-\frac{12}{35} h^{6}$,

Shkadov model (1.2), (1.4): $\mathscr{G}(h, c)=\frac{2}{5} q^{2}-\frac{4}{5} c q h+\frac{1}{3} c^{2} h^{2}$

$$
=\frac{2}{5} c^{2}-\frac{4}{15} c+\frac{2}{45}-\frac{2}{15} c^{2} h^{2},
$$

Model (1.2), (1.5):

$$
\begin{aligned}
\mathscr{G}(h, c) & =\frac{18}{35} q^{2}-\frac{34}{35} c q h+\frac{2}{5} c^{2} h^{2} \\
& =\frac{1}{35}\left[18 c^{2}+\frac{2}{3} c h-12 c-2 c^{2} h(h+1)+2\right] .
\end{aligned}
$$

The expression for $q$ given by (1.6) using (1.8) has been used to expand $\mathscr{G}$ when needed.

One-humped solitary wave solutions to (1.1), (1.3), (1.2), (1.4) and (1.2), (1.5) have been computed within the dynamical-systems setting described in the next section using the continuation software AuTO97 and its package HomCont for the computation of homoclinic orbits (Doedel et al. 1997). The speed $c$ and maximum height $h_{\mathrm{m}}$ of such waves are displayed in figure 1 as functions of $\delta$.

The turning point of the branch corresponding to Benney's equation signals the loss of solution for $\delta$ greater than $\delta^{\star} \approx 0.986$, a value that closely corresponds to the occurrence of blow-ups of unsteady solutions mentioned previously. By contrast, Ooshida's equation (1.3) and models (1.2), (1.4) and (1.2), (1.5) possess a one-humped solitary wave solution for all values of $\delta$, in agreement with what was obtained by Chang, Demekhin \& Kalaidin (1996) through integration of the much more cumbersome BL equations. While the asymptotic wave speed of order 2.5 reported in Chang et al. (1996) should be taken with care $\dagger$ the outcome of Ooshida's regularized

$\dagger$ The apparent good agreement between results from Shkadov's model and BL equations is probably fortuitous owing to limited streamwise resolution of the large- $\delta$ BL simulations (only 70 Fourier modes for strongly localized solitary waves). 
equation is clearly less satisfactory than the supposedly more reliable results from the two-equation models. Indeed, in the three cases the speeds of the solitary waves saturate but those obtained from (1.3) are a factor of more than 2 smaller than those obtained from models (1.2), (1.4) and (1.2), (1.5) or the BL results. The waves' maximum heights $h_{\mathrm{m}}$ also grow rapidly in the transition region around $\delta \sim 1.5-2$ and continue to increase beyond that range for the models and BL solutions, a trend which is not reproduced by Ooshida's solutions, suggesting that the shape of the waves is also not properly approximated by solutions to (1.3) at large $\delta$.

Two different flow regimes were distinguished by Ooshida (1999): the drag-gravity regime, which takes place for $\delta \ll 1$ and where the dynamics is governed by a balance between the viscous drag at the wall and gravity and surface tension, with inertia playing the role of a perturbation, and the drag-inertia regime at $\delta \gg 1$, when inertia effects are dominant. The rest of this note mainly focuses on the solitary-waves' asymptotic behaviour in the drag-inertia regime, using the tools of dynamical system theory.

\section{Solitary waves and dynamical system theory}

\subsection{General setting and drag-gravity regime}

Equation (1.9) can be recast as a three-dimensional dynamical system:

$$
U_{1}{ }^{\prime}=U_{2}, \quad U_{2}{ }^{\prime}=U_{3}, \quad U_{3}{ }^{\prime}=-3\left[\delta \mathscr{G}\left(U_{1}, c\right) U_{2}+\mathscr{H}\left(U_{1}, c\right)\right] / U_{1}^{3},
$$

in a phase space spanned by $\boldsymbol{U}=\left(U_{1}, U_{2}, U_{3}\right)$ where $U_{1}=h, U_{2}=h^{\prime}, U_{3}=h^{\prime \prime}$, and solutions to (1.9) are trajectories in that phase space.

Solitary wave solutions correspond to homoclinic orbits connecting fixed points to themselves. The fixed points of (2.1) are given by $U_{2}=U_{3}=0$ and

$$
3 \mathscr{H}\left(U_{1}, c\right)=\left(U_{1}-1\right)\left(U_{1}^{2}+U_{1}+1-3 c\right)=0,
$$

from which it is seen that $U_{1}=1$ is a solution arising from the scaling convention for $h$. Additional roots are given by

$$
U_{1}^{2}+U_{1}+1-3 c=0 .
$$

Accordingly, for $c>1 / 3$, i.e. for waves travelling faster than the average speed of the Nusselt flow as seen from (1.8), there is one supplementary positive solution:

$$
h_{\mathrm{II}} \equiv-1 / 2+\sqrt{3(c-1 / 4)},
$$

so that (2.1) then admits two fixed points $\boldsymbol{U}_{\mathrm{I}}=(1,0,0)$ and $\boldsymbol{U}_{\mathrm{II}}=\left(h_{\mathrm{II}}, 0,0\right)$. The study below extends the analysis developed by Pumir et al. (1983) for the Benney equation with $\delta \ll 1$ to the models introduced in $\S 1.1$ and $\S 1.2$. The case $\delta \gg 1$ is considered in the next subsection.

Let us first consider fixed point $\boldsymbol{U}_{\text {I }}$. The dispersion relation governing infinitesimal perturbations varying as $\exp (\lambda \xi)$ is

$$
\lambda^{3}+3 \delta \mathscr{G}(1, c) \lambda-3(c-1)=0 .
$$

Denoting the roots as $\lambda_{i}, i=1,2,3$, we have $\lambda_{1}+\lambda_{2}+\lambda_{3}=0$. Furthermore, one of the roots $\lambda_{1}$ is real and has the sign of the product $\lambda_{1} \lambda_{2} \lambda_{3}=3(c-1)$, thus is positive when $c>1$ and negative when $c<1$. The two others roots are complex conjugate (real) when

$$
\Delta_{\mathrm{I}}=4 \delta^{3} \mathscr{G}(1, c)^{3}+9(c-1)^{2}
$$




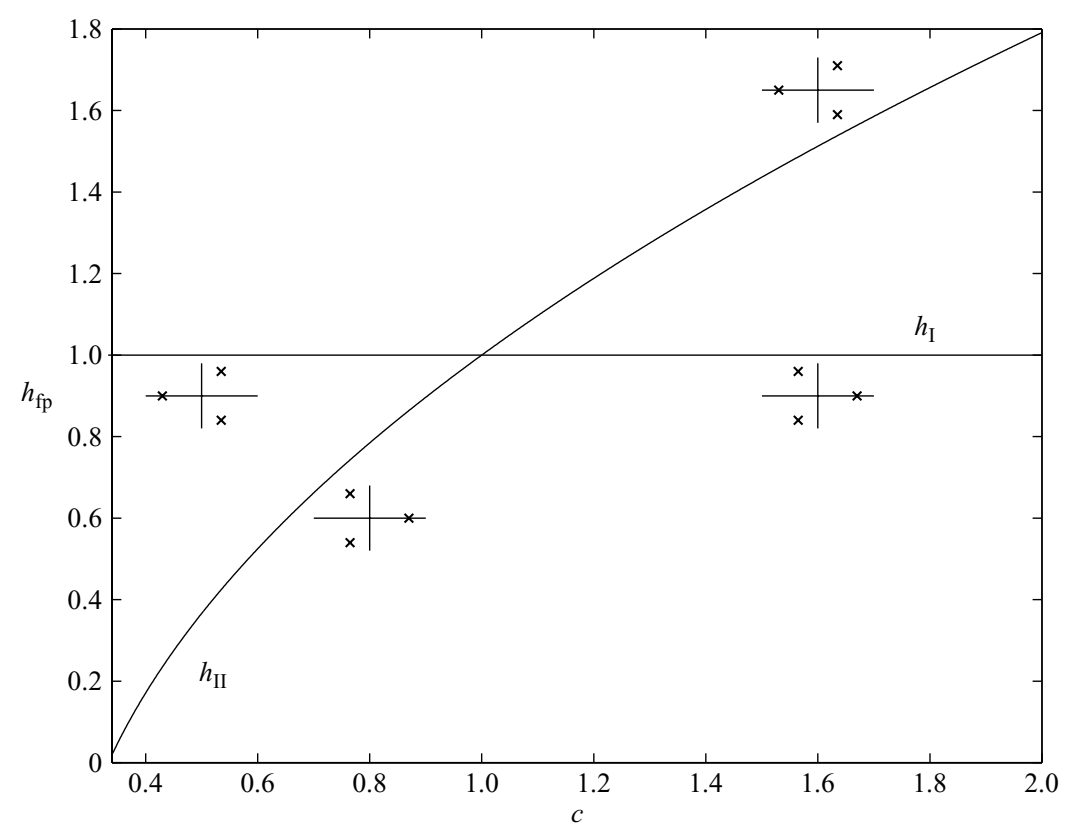

FIGURE 2. Locations of the fixed point height $h_{\mathrm{fp}}$ and stability diagram as function of the wave speed $c$ in the case of the Benney equation (1.1).

is positive (negative). In the same way, at the second fixed point $\boldsymbol{U}_{\text {II }}$, we obtain

$$
h_{\mathrm{II}}^{3} \lambda^{3}+3 \delta \mathscr{G}\left(h_{\mathrm{II}}, c\right) \lambda-3\left(c-h_{\mathrm{II}}^{2}\right)=0
$$

and the sum of the roots is again zero. One of roots is real and has the sign of $c-h_{\mathrm{II}}^{2}$, hence negative when $c>1$, since $c>h_{\mathrm{II}}$ implies $c-h_{\mathrm{II}}^{2}=c-\left(3 c-1-h_{\mathrm{II}}\right)=(1-c)+$ $\left(h_{\mathrm{II}}-c\right)<0$. By performing the transformation that allowed us to rescale the equation in order to reset $h_{\mathrm{II}}$ to one, it can be seen that the sign of

$$
\Delta_{\mathrm{II}}=4 \delta^{3} \mathscr{G}\left(h_{\mathrm{II}}, c\right)^{3}+9 h_{\mathrm{II}}^{3}\left(c-h_{\mathrm{II}}^{2}\right)^{2}
$$

is the same as that of $\Delta_{\mathrm{I}}$, which finishes to link the properties of $\boldsymbol{U}_{\text {II }}$ to those of $\boldsymbol{U}_{\mathrm{I}}$, just exchanging the dimensions of their stable and unstable manifolds.

The case of the Benney equation (Pumir et al. 1983) is the easiest one, thanks to the simplicity of the corresponding expression $\mathscr{G}(h, c)=\frac{2}{15} h^{6}$, independent of $c$. Since $\mathscr{G}$ is always positive, both fixed points have one real root and one complex pair for all $c$. As shown in figure 2, they are both saddle-foci. An exchange of properties is seen to take place at $c=1$, which makes the case degenerate with $h_{\mathrm{I}}=h_{\mathrm{II}}=1$. As proven by Gaspard (1993), the existence of homoclinic trajectories for the Benney equation with $c \approx 1$ when $\delta \ll 1$ stems from the perturbation of conditions $\Delta_{\mathrm{I}}=0$ and $c=1$ which define a codimension-two bifurcation at a double stationary-oscillatory instability with eigenvalues 0 , and $\pm \mathrm{i} \omega$. Since $\mathscr{G}(1, c)$ is positive, when $c>1$ we have $\lambda_{1}>0$ while $\lambda_{2,3}$ are complex conjugate with negative real values. The homoclinic orbit thus starts from $\boldsymbol{U}_{\mathrm{I}}$ along the one-dimensional unstable manifold $\mathscr{W}_{\mathrm{I}}^{u}$ in a monotonic way and returns to the fixed point by spiralling along the two-dimensional stable manifold $\mathscr{W}_{\mathrm{I}}^{s}$. At finite but small $\delta$ such an orbit can be understood as coming from the homoclinic bifurcation of a limit cycle arising from the Hopf bifurcation of $\boldsymbol{U}_{\text {II }}$ for $c>1$, then approaching and finally touching $\boldsymbol{U}_{\mathrm{I}}$ as its length is increased. Because $h_{\mathrm{II}}>h_{\mathrm{I}}=1$, the corresponding wave profile resembles a hump preceded by ripples 

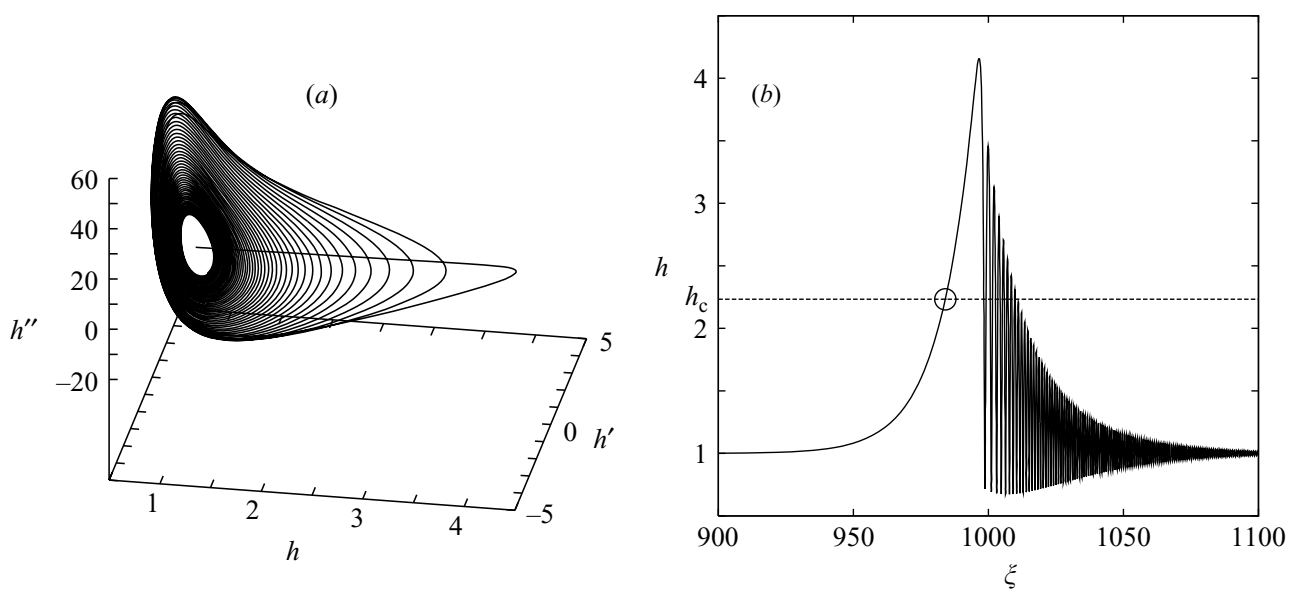

FIGURE 3. Typical solitary wave solution to model (1.2), (1.5) at large $\delta$, here $\delta=10$. (a) Trajectory in the phase space spanned by $\boldsymbol{U}=\left(U_{1}, U_{2}, U_{3}\right) \equiv\left(h, h^{\prime}, h^{\prime \prime}\right)$. The spiralling behaviour towards the fixed point $\boldsymbol{U}_{\mathrm{I}}$ has been truncated in order to point out the monotonic starting of the trajectory along $\mathscr{W}_{\mathrm{I}}^{u}$. (b) Profile of the wave $h=h(\xi)$ in the frame moving at speed $c$, with an indication of the critical level $h_{\mathrm{c}}$ to be defined in the text.

corresponding to the spiralling return to $\boldsymbol{U}_{\mathrm{I}}$ but the lack of homoclinic orbit for $\delta \sim 1$ remains unexplained by these considerations.

In the drag-gravity regime, the structure of homoclinic orbits corresponding to solitary waves is easily seen to follow directly from the analysis developed for the Benney equation since one can check that $\mathscr{G}(1, c)>0$ for the Ooshida equation, as well as for the two-equation models $(1.2,1.4)$ and $(1.2,1.5)$. Accordingly, $\Delta_{\mathrm{I}}$ is positive and the signatures of the fixed points are the same as for the Benney equation: fixed point $\boldsymbol{U}_{\mathrm{I}}$ is again a saddle-focus with a one-dimensional unstable manifold. Homoclinic orbits are then expected by continuity with the case of the Benney equation since the structure of the latter is recovered from these more elaborate models in the longwavelength limit.

\subsection{Asymptotic behaviour in the drag-inertia regime}

As can be seen from figure 1, the speeds of the one-humped solitary wave solutions to Ooshida's equation (1.3) and to the two-equation models (1.2), (1.4) and (1.2), (1.5) saturate as $\delta$ increases. This is precisely what we wish to predict from a direct analysis of the dynamical system in the drag-inertia regime, $\delta \gg 1$, while attempting to construct the corresponding homoclinic orbits. As seen in figure 3, these trajectories have three different parts: two extend the linearized dynamics around $\boldsymbol{U}_{\mathrm{I}}$ to the weakly nonlinear regime and the third, in-between, accounts for the strongly nonlinear region away from $\boldsymbol{U}_{\text {I }}$ where they bend back. In the course of our derivation, we will need only two empirical results: (i) that apparently smooth one-hump solitary waves do exist in the limit $\delta \rightarrow \infty$, with a monotonic rear and an oscillatory front, and (ii) that their speeds are larger than 1.

In the limit $\delta \rightarrow \infty$, the linearized dynamics around $\boldsymbol{U}_{\mathrm{I}}$ is controlled by (2.5). Setting $\lambda_{1}=2 \sigma$ and $\lambda_{2,3}=-\sigma \pm \omega$, we obtain

$$
\omega^{2}-3 \sigma^{2}=3 \delta \mathscr{G}(1, c) \quad \text { and } \quad 2 \sigma\left(\sigma^{2}+\omega^{2}\right)=3(c-1) .
$$

Assuming $c>1$ as suggested by the empirical results, and thus $\mathscr{G}(1, c)>0$, we have $\omega \sim \delta^{1 / 2} \sqrt{3 \mathscr{G}(1, c)}$ and $\sigma \sim \delta^{-1}(c-1) / 2 \mathscr{G}(1, c)$. Accordingly, the escape from $\boldsymbol{U}_{\mathrm{I}}$ along 
the one-dimensional unstable manifold $\mathscr{W}_{\mathrm{I}}^{u}$ is slow and monotonic while the convergence toward $\boldsymbol{U}_{\mathrm{I}}$ along its two-dimensional stable manifold $\mathscr{W}_{\mathrm{I}}^{s}$ happens to be a slow relaxation of fast oscillations, in full agreement with empirical results.

When the trajectory has left the immediate vicinity of $\boldsymbol{U}_{\mathrm{I}}$, one must return to the complete system. Focusing on the $\delta \gg 1$ range it may be preferable to rewrite (1.9) as

$$
\mathscr{G}(h, c) h^{\prime}=-\frac{1}{\delta}\left[\frac{1}{3} h^{3} h^{\prime \prime \prime}+\mathscr{H}(h, c)\right],
$$

with $\mathscr{H}(h, c)$ given by (1.10). In the inviscid limit, $\delta=\infty$, one finds $\mathscr{G}(h, c) h^{\prime}=0$, so that one can define the 'critical level' as the root in $h$ of

$$
\mathscr{G}\left(h_{\mathrm{c}}, c\right)=0 \text {, }
$$

at given $c$. This condition does not select the value of $c$, so let us return to the complete equation for $\delta<\infty$ in the vicinity of $h=h_{\mathrm{c}}$ whatever its value.

The homoclinic trajectory starts along the one-dimensional unstable manifold of $\boldsymbol{U}_{\text {I }}$, with linear eigenvalue $2 \sigma \sim \delta^{-1} \ll 1$. The dynamics along the unstable manifold can be studied by changing to the slow variable $\tilde{\xi}=\xi / \delta$, which leads to (2.9) being rewritten as

$$
\mathscr{G}(h, c) h^{\prime}=-\left[\mathscr{H}(h, c)+\frac{1}{3} \delta^{-3} h^{3} h^{\prime \prime \prime}\right],
$$

where the prime now denotes differentiation with respect to $\tilde{\xi}$. The last term in (2.11) is negligible along the first part of the trajectory corresponding to $\xi$ (or $\tilde{\xi}$ ) coming from $-\infty$, i.e. the rear of the wave. So, let us develop the argument at dominant order in $\delta$ :

$$
\mathscr{G}(h, c) h^{\prime}=-\mathscr{H}(h, c) .
$$

At given $c, \mathscr{G}(1, c)>0$ and $\mathscr{G}(h, c)$ decreases as $h$ increases, which is easily seen from the expressions given earlier in the three cases of interest $\dagger$. The dependent variable $h$ increases with $\tilde{\xi}$ as long as $h^{\prime}>0$. Since $\mathscr{H}(h, c)<0$ for $1<h<h_{\mathrm{II}}$ where $h_{\mathrm{II}}$ is given by (2.4), $h^{\prime}$ is positive as long as $h<h_{\mathrm{II}}$ and $h<h_{\mathrm{c}}$. If for the considered value of $c$, $h_{\mathrm{II}}<h_{\mathrm{c}}$, no singularity occurs and $h$ generically goes through a maximum, so that it cannot reach $h_{\mathrm{c}}$ at least in the rear part of the trajectory, which contradicts the assumption that we are considering one-hump solitary waves. On the other hand, if $h_{\mathrm{c}}$ is reached first, then a singularity takes place with $h^{\prime}$ diverging at $\tilde{\xi}=\tilde{\xi}_{\mathrm{c}}$, which now contradicts the assumption of smooth solitary waves derived from empirical evidence. The only possibility to remove the singularity is thus that $h_{\mathrm{II}}=h_{\mathrm{c}}$, in which case $\mathscr{G}$ and $\mathscr{H}$ are both zero for the same value of $h$, which selects the wave speed $c$ at dominant order in $\delta$.

Solving

$$
\mathscr{G}\left(h_{\mathrm{II}}, c\right)=0
$$

for $c$ with $h_{\text {II }}$ given by (2.4) yields the asymptotic values $c_{\infty}$ reached by $c$ when the limit $\delta \rightarrow \infty$ is taken. The values obtained for (1.3), (1.2), (1.4) and (1.2), (1.5) are

$$
\begin{array}{lll}
\text { Ooshida's equation (1.3): } & c_{\infty}=\frac{9}{841}(83+5 \sqrt{141}) & \approx 1.524, \\
\text { Shkadov's model }(1.2),(1.4): & c_{\infty}=1+1 / \sqrt{6}+\sqrt{1 / 2+\sqrt{2 / 3}} & \approx 2.556, \\
\text { Model (1.2), (1.5): } & c_{\infty}=\frac{1}{6}(9+\sqrt{43+2 \sqrt{37}}) & \approx 2.738,
\end{array}
$$

in good agreement with the value obtained from the numerics (figure $1 a$ ). Considering the Benney equation (1.1), condition (2.13) can never be achieved since $c$ is not present

$\dagger$ The result is immediate for Shkadov's model and straightforward for model (1.2), (1.5) since $c>1$ is assumed. For the Ooshida equation the decrease only occurs for $h^{2}>\frac{25}{27} c$ but this does not change the argument. 


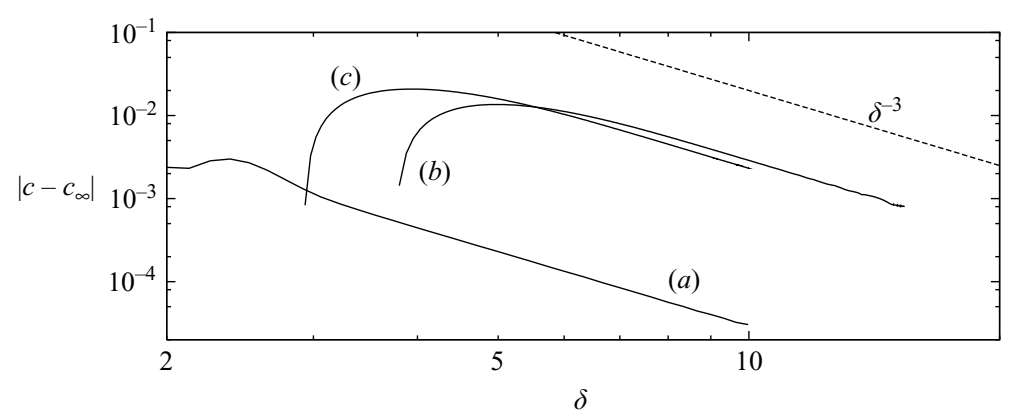

Figure 4. Convergence of the speed of solitary waves toward their asymptotic values $c_{\infty}$ as a function of $\delta$. (a) Ooshida's equation (1.3). (b) Shkadov's model (1.2), (1.4). (c) Model (1.2), (1.5).

in the expression for $\mathscr{G}$, which explains the lack of solitary wave solutions at large Reynolds numbers.

Now, when the limit $\delta \rightarrow \infty$ is not taken but $\delta$ just assumed to be large, the singularity in (2.11) remains at $h=h_{\mathrm{c}}$ defined by $\mathscr{G}(h, c)=0$. That singularity will be avoided again provided that the right-hand side is zero when $h=h_{\mathrm{c}}$. In the region $h \sim h_{\infty}$ where $h_{\infty}=h_{\mathrm{II}}(\delta \rightarrow \infty)$ as determined above, the shape of the solution has no reason to change rapidly as $\delta$ increases so that one can generically expect $h^{\prime \prime \prime} \sim h_{\infty}^{\prime \prime \prime}$, where $h_{\infty}^{\prime \prime \prime} \neq 0$ is the asymptotic value of the third derivative of $h$ in $\tilde{\xi}$ (the slow variable). The condition replacing (2.13) is thus

$$
\mathscr{G}\left(h_{\mathrm{c}}(\delta), c(\delta)\right)=K \delta^{-3}
$$

where $K$ is a numerical constant depending on $h_{\infty}$ and $h_{\infty}^{\prime \prime \prime}$. Looking for the solution to equations $(2.10)$ and (2.14) through their expansion around $\left(h_{\infty}, c_{\infty}\right)$ yields

$$
c-c_{\infty} \propto \delta^{-3}
$$

a convergence rate which is verified well by the numerics as shown in figure 4.

While explaining the asymptotic behaviour of the speed of one-hump solitary waves, the argument says nothing about how the trajectory bends back towards $\boldsymbol{U}_{\mathrm{I}}$, so it cannot justify their existence that has thus to be taken for granted. This existence property is likely to be more difficult to prove than in the small- $\delta$ range where one can make use of Gaspard's result. In this respect, it should be noted that the critical value $h_{\mathrm{c}}$ introduced in the derivation makes sense only on the slow rear part of the wave, i.e. for the value of $\tilde{\xi}$ which achieves the critical condition for the first time when increasing from $-\infty$, since $h=h_{\mathrm{c}}$ also occurs at least once in the fast oscillating front part when $h$ decreases from its maximum value $h_{m}>h_{\mathrm{c}}$. However the dominant term in the equation is then $h^{\prime \prime \prime}$ and to deal with it one has to turn to an expression for the dynamics in terms of a fast variable $\hat{\xi}=\xi \sqrt{\delta}$ and no singularity occurs when $h \sim h_{\text {c }}$. The third derivative term is far from singular as long as $h$ is bounded away from zero. Were this no longer the case, steady-state waves would no longer exist and time-dependent solutions would experience blow-up, but this is irrelevant to the models considered here, as inferred from computational evidence.

\section{Summary and conclusion}

We have considered one-humped solitary wave solutions to one-equation models (1.1) and (1.3), and depth-averaged two-equation models (1.2), (1.4) and (1.2), (1.5) 
using the tools of dynamical system theory. These solutions all derive from similar dynamical systems, differing one from another by the expression for the inertial terms. Analytic expressions for their speed $c_{\infty}$ have been obtained for the three last models and the expected cubic convergence $c-c_{\infty} \propto \delta^{-3}$ has been verified. The lack of onehumped solitary waves at large $\delta$ for the Benney equation (1.1) has been attributed to the absence of freedom in the expression for $\mathscr{G}$ to match condition (2.13).

In view of comparisons with experiments, the wave profile with fast oscillations preceding the hump shown in figure 3 may seem unrealistic, while such oscillations are known to be strongly damped by viscous dispersion effects. It is thus essential to observe that the result obtained here in the restricted case of films over vertical planes without viscous dispersion extends to the general case, provided that a single equation, of possibly higher order in time, can be obtained through the procedure leading to (1.9). Models derived by us (Ruyer-Quil \& Manneville 2000) have been shown to yield results in good agreement with experiments at moderate Reynolds numbers for which two-dimensional solitary waves are indeed observed. This validation is therefore an important supplementary step towards the theoretical understanding of the dynamics of surface waves and the secondary patterning of flows over inclined planes.

\section{REFERENCES}

Alekseenko, S. V., Nakoryakov, V. Y. \& Pokusaev, B. G. 1994 Wave Flow of Liquid Films. Begell House.

Benney, D. J. 1966 Long waves on liquid films. J. Math. Phys. 45, 150-155.

Chang, H.-C., Demekhin, E. \& Kalaidin, E. 1996 Simulation of noise-driven wave dynamics on a falling film. AIChE J. 42, 1553-1568.

Doedel, E. J., Champneys, A. R., Fairgrieve, T. F., Kuznetsov, Y. A., Sandstede, B. \& Wang, X.-J. 1997 Auto97: Continuation and bifurcation software for ordinary differential equations. Tech. Rep.. Department of Computer Science, Concordia University, Montreal, Canada, (Available by FTP from ftp.cs.concordia.ca in directory pub/doedel/auto).

Gaspard, P. 1993 Local birth of homoclinic chaos. Physica D 62, 94-122.

KapitZA, P. 1948 Wave flow of thin viscous fluid layers. Zh. Ekper. Teor. Fiz. 18, 3-28 (English transl. in Collected Papers of P. L. Kapitza (ed. Ter Haar), pp. 662-689, Pergamon, 1965).

KAPITZA, P. \& KapITZA, S. 1949 Wave flow of thin layers of a viscou fluid. Zh. Ekper. Teor. Fiz. 19, 105-120 (English transl. in Collected Papers of P. L. Kapitza (ed. Ter Haar), pp. 690-709, Pergamon, 1965).

LiU, J. \& Gollub, J. P. 1994 Solitary wave dynamics of film flows. Phys. Fluids 6, 1702-1712.

Malamataris, N. A., Vlachogiannis, M. \& Bontozoglou, V. 2002 Solitary waves on inclined films: Flow structure and binary interactions. Phys. Fluids 14, 1082-1094.

OoshidA, T. 1999 Surface equation of falling film flows which is valid even far beyond the criticality. Phys. Fluids 11, 3247-3269.

Pumir, A., Manneville, P. \& Pomeau, Y. 1983 On solitary waves running down an inclined plane. J. Fluid Mech. 135, 27-50.

Rosenau, P., Oron, A. \& Hyman, J. 1992 Bounded and unbounded patterns of the Benney equation. Phys. Fluids A 4, 1102-1104.

Ruyer-Quil, C. \& Manneville, P. 2000 Improved modeling of flows down inclined planes. Eur. Phys. J. B 15, 357-369.

Ruyer-Quil, C. \& Manneville, P. 2004 Comment on "low-dimensional models for vertically falling viscous films". Phys. Rev. Lett. 93 (19), 199401.

Shkadov, V. 1967 Wave flow regimes of a thin layer of viscous fluid subject to gravity. Izv. $A k$. Nauk SSSR, Mekh. Zhid. Gaza 2, 43-51 (English transl. in Fluid Dyn. 2, 29-34).

SHKadov, V. 1977 Solitary waves in a layer of viscous liquid. Izv. Ak. Nauk SSSR, Mekh. Zhid. Gaza 1, 63-66.

Yıн, C. S. 1955 Stability of two-dimensional parallel flows for three-dimensional disturbances. Q. Appl. Maths 12, 434. 